\title{
LAUDATIO JUBILACIÓN FERNANDO DÍEZ RODRÍGUEZ
}

\author{
Pedro Ruiz Torres
}

Universitat de València

\section{FERNANDO DÍEZ, EL HISTORIADOR DISCRETO}

Hace aproximadamente un año, en el acto más bien de homenaje que de despedida a varios profesores de la Facultad de Geografía que acababan de jubilarse, me encargué de la lautatio del profesor Fernando Díez. Lo hice de un modo bastante informal, con la mayor espontaneidad posible, porque era consciente de lo incómodo que se siente Fernando cuando alguien habla de sus méritos, reacio como suele ser a los elogios si se trata de su persona. Puse como ejemplo de ese rechazo el correo electrónico que me había enviado poco antes, después de haberle pedido que destacara algo de su vida académica:

Ahí va una somera información. Filosofía y Letras. Especialidad de Filosofía (Universidad de Comillas, Madrid). Idem. Especialidad de Historia y Geografía (Universidad de Valencia).

Universidad Laboral de Cheste. Profesor de enseñanzas medias desde 1972 hasta 1989.

Profesor Universidad de Valencia, desde 1989 hasta septiembre 2017.

Lo demás queda a tu discreción

Hasta el jueves

Fernando.

"Discreción", según el diccionario de la RAE, significa "sensatez para formar juicio y tacto para hablar u obrar", "don de expresarse con agudeza, ingenio y oportunidad", "reserva, prudencia, circunspección". La sensatez es también prudencia o cautela para guardar un secreto, para contar lo que se sabe y no hay necesidad de que conozcan los demás. Supongamos que seguía al pie de la letra semejante recomendación, ¿qué es lo que se sabía y no había necesidad de comentar sobre su trayectoria de historiador? Fernando tiene una nula inclinación a ponerse a sí mismo en el centro de las conversaciones, a hablar de sus méritos como profesor o como investigador. Su actitud, en ese sentido, poco frecuente en nuestro entorno, me planteó desde el principio la cuestión de que había demasiadas cosas importantes que muchos de los presentes en aquel acto seguramente desconocían. Si para colmo debía hacerlo con el tacto 
y la sensatez a que me obligaba su presencia, lo mejor era huir de las alabanzas, no caer en los tópicos de la clásica laudatio. En vez de leer un texto previamente redactado, improvisaría sobre la marcha y estaría más pendiente del público y sobre todo de la reacción del interesado. Me temo ahora que el pharmarkon del discurso escrito, que sirve de remedio a la pérdida de la memoria de aquel acto celebrado hace más o menos un año, contenga a cambio el veneno que impide rescatar el recuerdo auténtico de lo vivido. Mi intervención oral sintonizó mejor de lo que puede hacer este texto con la personalidad de alguien tan poco dado al protocolo y al que le importa mucho la ponderación en el juicio y todo lo demás que es propio de la discreción.

Fernando Díez se incorporó como alumno a la Universidad de Valencia durante el curso académico 1970-71, es decir, justo cuando se inauguró el edificio en el que hace un año tuvo lugar el mencionado acto. Ahora este edificio es la sede de la Facultad de Geografía e Historia, pero a principios de los setenta daba cabida a las tres secciones (Historia y Geografía, Filosofía y Lengua y Literatura) de la Facultad de Filosofía y Letras. Antes, en la Universidad de Comillas, Fernando se había licenciado en Filosofía y Letras, especialidad de Filosofía, por lo que en Valencia se disponía a hacer otro tanto, pero en la sección de Historia y Geografía. Desde octubre de 1970 hasta junio de 1973, tres años en los que se recrudeció la represión del régimen de Franco, Fernando hizo compatibles los estudios de cara a esta segunda especialización, el compromiso contra la dictadura y la participación en no pocos de los seminarios, la mayoría de ellos sobre marxismo, que tenían lugar fuera de la universidad y al margen de la vida académica. No creo ni mucho menos ser el único de entre sus compañeros de curso durante esos tres años que guarde un vivo recuerdo de su persona a principios de los setenta al menos por dos motivos. El primero es que Fernando había leído a Marx, a Engels y a otros pensadores de relieve en profundidad y con sentido crítico, en vez de hablar de oídas o repetir las simplificaciones y tergiversaciones de las ideas de los supuestos fundadores del marxismo que eran moneda común en el medio universitario de izquierdas. Además Fernando se significaba por su forma pausada y concisa de hablar un castellano cuidado y con acento del norte, además de por su figura y su barba un tanto quijotescas. Él y su compañera, Charo Alonso, formaban una pareja inseparable de cuya compañía y conversación era posible disfrutar en circunstancias diversas, en aquellos años difíciles en los que la opinión política y la voluntad de saber se reforzaban mutuamente.

Al terminar en 1973 su segunda especialización, Fernando fue contratado como profesor de enseñanza media en la Universidad Laboral de Cheste. Las Universidades Laborales, dependientes durante muchos años del Ministerio de Trabajo, habían sido promovidas por José Antonio Girón de Velasco sobre la base de la ideología falangista, pero tras la llegada al poder de los tecnócratas 
fueron perdiendo su impronta inicial durante la década de los sesenta. En 1969, en un extenso terreno de más de un millón y medio de metros cuadrados, a treinta kilómetros de la ciudad de Valencia, se creó la Universidad Laboral de Cheste, con capacidad para cinco mil alumnos en régimen de internado. Su función específica era proporcionar una orientación y una preparación básica a niños de 11 a 14 años de edad, hijos de familias trabajadoras con pocos recursos, que recibían becas de estudio para incorporarse al bachillerato especial de carácter profesional que en 1949 el régimen de Franco había puesto en marcha. En una primera etapa, la Universidad de Cheste impartió cursos de orientación y el bachillerato elemental, por lo que sus alumnos al terminar debían irse a otras Universidades Laborales si querían proseguir sus estudios. Tras la aprobación en 1969 de la Ley General de Educación de Villar Palasí, las Universidades Laborales pasaron a depender del Ministerio de Educación y la de Cheste se encargó de la primera y la segunda etapa de la EGB, de la Formación Profesional, del Bachillerato Unificado Polivalente y del Curso de Orientación Universitaria. En 1973, poco después de iniciarse este nuevo periodo, Fernando Díez entró como profesor en dicho centro, en unos años que Javier Ignacio Chust Torrent, un antiguo alumno, recuerda en su libro La Universidad Laboral de Cheste (1969-1978) precursora de la innovación educativa en España, publicado por la editorial Tirant Humanidades en 2016, con estas palabras: "Creo firmemente que existieron en este Centro Docente aspectos que necesitan ser conocidos por la sociedad actual, y más concretamente por los que nos dedicamos al mundo de la enseñanza y de la educación". Desde un punto de vista pedagógico, didáctico y metodológico, añade el citado autor, "supusieron un antes y un después en la innovación educativa en nuestro país".

Si hago hincapié en ello es porque fue una etapa muy importante en la trayectoria de Fernando Díez. Desde entonces, la docencia no dejó de ocupar el primer plano de su labor intelectual. La enseñanza siempre tuvo para él un enorme valor, a diferencia de tantos otros que huyen de ella como si fuera una carga. Preparaba concienzudamente sus cursos, buscaba una y otra vez la manera de trasmitir conocimientos de modo claro y accesible, con el apoyo de recursos didácticos bien elegidos, en función de los contenidos de cada una de las materias y de los tipos de estudiantes. Durante las cuatro décadas en que fue profesor de la Universidad de Valencia, desde 1987 -y no desde 1989 como me puso en su correo electrónico- hasta 2017, primero en la Escuela de Trabajo Social, más tarde en la Facultad del mismo nombre y asimismo cuando se incorporó a la docencia de la asignatura "Culturas del Trabajo y Políticas Sociales en los siglos XIX y XX", en el Máster de Historia Contemporánea, sus alumnos recibieron esa enseñanza verdaderamente de calidad que tantas veces echaron en falta. Su jubilación trae así una pérdida insustituible, por lo que deberíamos 
lamentar que no exista en nuestras universidades alguna forma de conservar un cierto vínculo con este tipo de profesores. Ello sin duda redundaría en beneficio de la formación de los alumnos, que ha de ser lo que más nos importe.

Para desmentir una vez más a aquellos que contraponen la calidad de la docencia a la excelencia en la investigación y reforzar por el contrario la idea de que una y otra son perfectamente compatibles, Fernando Díez destacó pronto en este otro ámbito. Sin la intención de hacer méritos como profesor universitario, por cuanto seguía siendo docente en Cheste y asumía cargos cada vez de mayor responsabilidad en la dirección y organización de ese Centro de Enseñanzas Integradas (la nueva denominación, a partir de 1979, de la antigua Universidad Laboral de Cheste), en 1980 vio la luz su primer libro Prensa agraria en la España de la Ilustración. El Seminario de Agricultura y Artes dirigido a los Párrocos (1797-1808). Publicado en Madrid por el Ministerio de Agricultura, en una colección de estudios que ganó prestigio por su valiosa contribución a la historia agraria, los resultados de la aludida investigación modificaron sustancialmente la idea demasiado simple y negativa que teníamos de la época de Godoy. El trabajo se había iniciado tiempo atrás. En la dedicatoria de este libro, su autor agradece al profesor Antonio Mestre que le hubiera puesto sobre la pista de este semanario completamente desconocido. La investigación tomó cuerpo primero en su tesis de licenciatura y prosiguió sin prisas, hasta convertirse en una obra que continúa siendo de obligada consulta para los estudiosos del siglo XVIII en España. Tras ella vinieron tres aportaciones muy diferentes en la década de los ochenta, que confirmaron y una vez más pusieron de relieve los rasgos característicos de su modo de investigar.

Fernando Díez tiene ideas muy claras acerca de lo que merece la pena convertirse en objeto de estudio y sobre la metodología a emplear: acierta a la hora de encontrar fuentes primarias de interés en los archivos, muchas de ellas nunca utilizadas antes; analiza de modo crítico y contrastado la información obtenida tras la consulta de la documentación; utiliza la narración, pero no de un modo meramente descriptivo, a la manera tradicional, sino con el fin de explicar lo sucedido y hacerlo comprensible. Además, él no se sentía entonces presionado por la urgencia de engrosar un curriculum de profesor universitario. Al placer por el trabajo erudito se añadía la plena conciencia de que era preciso interpretar los datos y asimismo transmitir a otros -fueran o no especialistas- los conocimientos obtenidos por medio de una escritura cuidada y precisa. Tanto en su primer libro, como en los otros tres trabajos que aparecieron en la década de los ochenta, los rasgos anteriores se manifestaron con creces. Uno de esos trabajos posteriores a su primer libro estuvo dedicado a la recepción del liberalismo económico en la prensa ilustrada y en 1981 fue publicado en las actas del coloquio celebrado en Lisboa sobre liberalismo en la Península Ibérica en 
la primera mitad del siglo XIX, organizado por Miriam Halpern Pereira, en el que entre otros historiadores de mucho prestigio participaron Pierre Vilar y Antonio Manuel Hespanha. En 1984 su artículo titulado "Los papeles del correjero", un excelente ejercicio de microhistoria, vio la luz en el número 5 de la revista Estudis d'història contemporànea del País Valencià, en un monográfico dedicado a la crisis del Antiguo Régimen. En 1988 apareció su estudio introductorio a la edición facsímil, por parte del Ministerio de Agricultura, Pesca y Alimentación, de El Correo General de España, un semanario de 1770 y 1771 creado y dirigido por Mariano Nipho. Nipho es un personaje muy interesante en el que, como se resalta en el estudio preliminar, por primera vez se encarnó en España la figura del periodista profesional.

En septiembre de 1987 una serie de circunstancias, poco previsibles años atrás, hicieron que Fernando Díez fuera contratado como profesor asociado a tiempo parcial, mientras seguía en la Escuela de Formación del Profesorado de E.G.B en Cheste. Dos años después, en octubre de 1989, dejó su antigua ocupación y se incorporó como profesor con dedicación completa al departamento de Historia Contemporánea de la Universidad de Valencia, para dar clases en la Escuela de Trabajo Social. No por ello cambió su manera de concebir la investigación del modo que hemos visto hace un momento, si bien durante algunos años la temática se fue desplazando del mundo agrario al artesanal y se concentró en el ámbito de la ciudad de Valencia durante el siglo XVIII. Unos cuantos artículos en diversas revistas de reconocido prestigio (Recerques, Estudios de Historia Social, Historia Social, Revista de Historia Económica) dieron a conocer una pequeña parte de los resultados de su investigación con vistas a la tesis doctoral. La tesis, que obtuvo la máxima calificación, fue juzgada por un tribunal presidido por Jordi Nadal y del que también formaron parte Josep Fontana, Jaume Torras, José Manuel Pérez García y Jesús Millán. Los artículos -sobre el nivel de vida de los trabajadores, la estructura ocupacional, la crisis gremial y los problemas de la sedería o el sistema benéfico asistencial en una ciudad preindustrial como era Valencia en el setecientos- vinieron en compañía de un libro, titulado Viles y mecánicos. Trabajo y sociedad en la Valencia preindustrial (València, Edicions Alfons el Magnànim, 1990,) en el que Fernando vertió y reelaboró la mayor parte del trabajo que le había servido para obtener el título de doctor en Historia por la Universidad de Valencia.

La última década de la pasada centuria fue la de su consolidación como docente y como investigador en el seno de nuestra institución, primero como Profesor Titular de Escuela Universitaria, después en calidad de Catedrático de Escuela Universitaria. También ese final de siglo trajo un cambio de rumbo en su investigación, que sin dejar de interesarse por las condiciones materiales de vida y de trabajo en el mundo artesanal del setecientos, empezó a hacerlo también por la apología del lujo y en definitiva del hombre consumidor en el 
pensamiento ilustrado, por las ideas sobre el trabajo en la obra de Antonio de Capmany y por el sistema benéfico-asistencial en la Valencia del siglo XIX. Con el inicio del siglo XXI dicho cambio de orientación se hizo patente y desbordó el ámbito valenciano y del conjunto de España. Su artículo "El discurso sobre el trabajo en el Siglo de las Luces", publicado en 2001 en el número 42 de la revista Sociología del Trabajo, lo puso de relieve y sobre todo su libro Utilidad, deseo y virtud. La formación de la idea moderna de trabajo, editado por Península en 2001. De nuevo iba a salir a relucir la originalidad y la madurez de una investigación llevada a cabo minuciosamente y sin precipitación, con criterio propio e indiferente a la moda del momento. La continuación de este libro no tardaría en llegar. En 2006 el Servei de Publicacions de la Universitat de València editó El trabajo transfigurado. Los discursos del trabajo en la primera mitad del siglo XIX. Ocho años más tarde todo ello culminó en una obra de envergadura, destinada a perdurar y a convertirse en uno de los libros de historia de las ideas más importantes que han aparecido en los últimos años. Me refiero a Homo Faber. Historia intelectual del trabajo, 1675-1945, editado en 2014 por Siglo XXI.

Las publicaciones de Fernando Díez no se agotan ni mucho menos en los libros y los artículos mencionados, pero no pretendo ser exhaustivo y me limitaré a añadir algunos temas por los que él también se ha interesado: la figura de E. P. Thompson y la crisis del marxismo, la literatura de la condición obrera y el lenguaje de la proletarización, "el trabajador de carácter" en el sentido de Richard Sennett, la controvertida relación entre pobreza, trabajo y asistencia, la previsión social etc. Su último libro -que dentro de poco dejará de serlo, porque hay otro a punto de aparecer- es un sugerente y provocativo ensayo La imaginación socialista. El ciclo histórico de una tradición intelectual, que en 2016 publicó la editorial Siglo XXI. Contiene una crítica de la izquierda, tal y como hoy se nos presenta, y deberíamos tenerla muy en cuenta en momentos como estos de desconcierto y de falta de ideas para hacer posible un futuro mejor.

Docencia e investigación a partes iguales, en un equilibrio ejemplar y poco frecuente, han sido las dos actividades a las que se ha dedicado Fernando Díez desde que en junio de 1973 terminó sus estudios de Historia y Geografía en la Facultad de Filosofía y Letras de la Universidad de Valencia, hace por tanto cuarenta y cinco años. Durante su etapa de profesor de enseñanza media asumió funciones relevantes de gestión y de dirección, pero debió haber quedado colmado porque en ningún momento le tentó hacer algo parecido en el ámbito universitario. No obstante, antes de poner punto y final a esta laudatio, me gustaría aclarar que su manera de concebir la docencia y la investigación en historia va mucho más allá del mero ejercicio de una profesión o de una actividad exclusivamente académica. Que se haya curado de la ingenuidad que muchos tuvimos hace bastantes años, al dar por descontado que de la unión de la teoría 
y la praxis saldría forzosamente una sociedad más justa e igualitaria, no significa que renuncie a que esto último sea un horizonte de futuro siquiera a largo plazo. Fernando Díez antepone la inclinación por el trabajo bien hecho y el reconocimiento de la valía intelectual a cualquier otra cosa, tal vez porque considera que se trata de condiciones necesarias para pensar con fundamento y con sentido de la realidad en cómo hacer posible una sociedad mejor. Tanto su voracidad de lector, que continuamente desborda los límites convencionales de las disciplinas y de los periodos históricos, como la originalidad de su trabajo de historiador, constituyen el marco en el que Fernando Díez inserta su preocupación por lo que nos ocurre y su crítica de lo que no le gusta. Fernando Díez es una persona moderada, sin excesos, en definitiva un hombre discreto.

Así termina su último libro, La imaginación socialista, y con ello también mi intervención:

Quizá la nueva imaginación crítica tenga que empezar, como ocurrió con la socialista, por alguna forma de utopismo, experimentalismo y ensayismo con el fin de escrutar, sin limitaciones, los contornos de los problemas existentes y la posibilidad de avanzar una respuesta. También para introducir una nota de ilusión y de esperanza. En cualquier caso, tenemos que recuperar el interés por el espectro más amplio posible de temas y problemas que debieran ser objeto de escrutinio, sin someter la mirada a algún canon de asuntos amortizados en lo que todavía pretendería pasar por una sensibilidad de izquierdas o simplemente socialista. Como si la nueva imaginación crítica pudiera contentarse con revitalizar la preocupación por los recortes de las políticas sociales, la pobreza, la distribución de la riqueza, la precarización del empleo y cosas parecidas, prevaleciéndose de una posición puramente defensiva y adocenándose sistemáticamente en la misma. Como si no hubiese toda otra serie de fenómenos de todo tipo de la más candente actualidad que se ciernen sobre nosotros de manera inquietante y sobrecogedoras, nos afectan en nuestra vida, también en la más cotidiana, y a los que tendemos, sin embargo, a someternos pensando que, después de todo, gozan del marchamo de la exaltada e inmoderada modernidad con que se presentan.

\section{PUBLICACIONES DE FERNANDO DÍEZ RODRÍGUEZ}

\section{Revistas}

"El nivel de vida de la población trabajadora en la ciudad preindustrial: condiciones para una difícil subsistencia, Valencia, siglo XVIII", en Estudios de Historia Social de España, vol. III y IV (1989), pp. 53-69.

"Los papeles del correjero", en Estudis d'historia contemporánea del País Valencià, vol. 5, nº 5, (1984), pp. 245-274. 
"L'Estructura ocupacional d'una ciutat pre-industrial, València, segle XVIII", en Recerques: Història, economia i cultura, no 24 (1991), pp. 75-90.

"La crisis gremial y los problemas de la sedería valenciana (finales del siglo XVIII y principios del siglo XIX)", en Revista de Historia Económica, vol. X, nº 1 (1992), pp. 39-61.

"Estructura social y sistema de benéfico-asistencial en la ciudad preindustrial", en Historia Social, no 13 (1992), pp. 101-121.

"La apología ilustrada del lujo en España. Sobre la configuración del hombre consumidor", en Revista Social, no 37 (2001), pp. 3-25.

"El gremialismo de Antonio de Capmany (1742-1813). La idea de trabajo de un conservador ingenuo", en Historia y Política, vol. 2001/1, n 1 (2001), pp. 171-206.

"El discurso del trabajo en el Siglo de las Luces", en Sociología del Trabajo, no 42 (2001), pp. 119-145.

"El trabajo y la vida. Una perspectiva histórica", en Empresa y Humanismo, vol. X, $n^{\circ} 1 / 07$ (2007), pp. 35-81.

"Trabajo, pobreza y beneficencia. En torno a las \&quot;Memorias sobre el Pauperismo\&quot; de Alexis de Tocqueville", en Historia y Política, vol. Enero/Junio, no 19 (2008), pp. 201-230.

"La formación de la clase obrera en Inglaterra: E.P. Thompson y la crisis del Marxismo", en Sociología Histórica, vol. 3, n 3 (2013), pp. 251-284.

Libros

Prensa agraría en la España de la Ilustración. El Semanario de Agricultura y Artes dirigido a los párrocos (1797-1808), Ministerio de Agricultura, Pesca y Alimentación. Series Estudios (1980), 239 p.

Viles y Mecánicos. Trabajo y sociedad en la Valencia preindustrial, Edicions Alfons el Magnànim - IVEI (1990), 197 p.

La sociedad desasistida. El sistema benéfico-asistencial en la Valencia del siglo XIX, Publicaciones de la Diputación de Valencia. Historia Local (1993), 269 p.

Utilidad, deseo y virtud. La formación de la idea moderna del trabajo, Península (2001), 303 p.

El trabajo transfigurado. Los discursos del trabajo en la primera mitad del siglo XIX, Servei de Publicacions de la Universitat de València (2005), 308 p.

La crisis gremial y la organización de la producción y del trabajo en la sedería valenciana (finales del siglo XVIII y principios del siglo XIX), Libros de la Catarata (1996), pp. 134-152.

La literatura de la "condición obrera" y el lenguaje de la proletarización, Biblioteca Nueva (2005), pp. 71-102.

Pobreza, trabajo y asistencia. Consideraciones históricas de una relación polémica, Editorial Bormarzo (2008), pp. 17-52.

La previsión social en la España del siglo XVIII. Realidad, reforma e historia comparada, Siglo XXI (2009), pp. 67-98. 


\section{Contribuciones a Congresos}

La recepción del liberalismo económico en la prensa ilustrada, 1797-1808. Comunicación. Congreso: O Liberalismo na Península Ibérica na primeira metade do sécolo XIX, Lisboa (Portugal), 1981.

La población de la ciudad de Valencia en el siglo XVIII. Censos y Vecindarios. Comunicación. Congreso: Primeres Jornades d'estudi sobre la població del País Valencià, Valencia (España), 1986.

La formación del discurso del trabajo proletarizado en la primera mitad del siglo XIX. Ponencia. Congreso: The Skilled Worker: Between Guild and Resistence, Castellón (España), 2003.

La previsión social en la España del siglo XVIII. Realidad, reforma e historia comparada. Ponencia invitada. Congreso: La previsión social en la historia. VI Congreso de Historia Social. Vitoria, 2008, Madrid (España), 2009.

Organización de congresos, seminarios, jornadas, etc., científico-tecnológicos

Empleo y exclusión social: Rentas Mínimas y otros mecanismos de inserción sociolaboral. Investigación. Internacional, 2004. 
